\title{
Influences of Lead (II) Chloride on the Nitrogen Metabolism of Spinach
}

\author{
Xiao Wu • Chao Liu • Chunxiang Qu • Hao Huang • \\ Xiaoqing Liu • Liang Chen • Mingyu Su • Fashui Hong
}

Published online: 23 June 2009

(C) Humana Press Inc. 2009

\section{Erratum to: Biol Trace Elem Res (2008) 121:258-265 \\ DOI 10.1007/s12011-007-8046-y}

The original version of this article unfortunately contained a mistake. The authors' first name and last name were interchanged in the original article. The correct names are shown above.

The online version of the original article can be found at http://dx.doi.org/10.1007/s12011-007-8046-y

X. Wu $\cdot$ C. Liu $\cdot$ C. Qu $\cdot$ H. Huang $\cdot$ X. Liu $\cdot$ L. Chen $\cdot$ M. Su $\cdot$ F. Hong $(\bowtie)$

College of Life Sciences, Suzhou University, Suzhou 215123, People's Republic of China

e-mail: Hongfsh_cn@sina.com 